\title{
Establishment of an inducing medium for type III effector secretion in Xanthomonas campestris pv. campestris
}

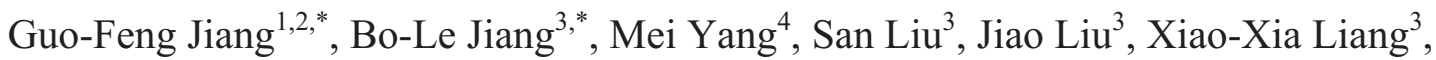 \\ Xian-Fang $\mathrm{Bai}^{3}$, Dong-Jie Tang ${ }^{3}$, Guang-Tao Lu ${ }^{3}$, Yong-Qiang $\mathrm{He}^{3}$, Di-Qiu Yu ${ }^{1}$, \\ Ji-Liang Tang $^{3}$ \\ ${ }^{1}$ Key Laboratory of Tropical Forest Ecology, Xishuangbanna Tropical Botanical Garden, \\ Chinese Academy of Sciences, Kunming, Yunnan, China. \\ ${ }^{2}$ Graduate School of Chinese Academy of Sciences, Beijing, China. \\ ${ }^{3}$ State Key Laboratory for Conservation and Utilization of Subtropical Agro-bioresources, \\ The Key Laboratory of Ministry of Education for Microbial and Plant Genetic Engineering, and College \\ of Life Science and Technology, Guangxi University, Nanning, Guangxi, China. \\ ${ }^{4}$ School of Chemistry and Chemical Engineering, Guangxi University, Nanning, Guangxi, China.
}

Submitted: January 1, 2012; Approved: September 10, 2012.

\begin{abstract}
It is well known that the type III secretion system (T3SS) and type III (T3) effectors are essential for the pathogenicity of most bacterial phytopathogens and that the expression of T3SS and T3 effectors is suppressed in rich media but induced in minimal media and plants. To facilitate in-depth studies on T3SS and T3 effectors, it is crucial to establish a medium for T3 effector expression and secretion. Xanthomonas campestris pv. campestris $(X c c)$ is a model bacterium for studying plant-pathogen interactions. To date no medium for Xcc T3 effector secretion has been defined. Here, we compared four minimal media (MME, MMX, XVM2, and XOM2) which are reported for T3 expression induction in Xanthomonas spp. and found that MME is most efficient for expression and secretion of Xcc $\mathrm{T} 3$ effectors. By optimization of carbon and nitrogen sources and $\mathrm{pH}$ value based on MME, we established XCM1 medium, which is about 3 times stronger than MME for Xcc T3 effectors secretion. We further optimized the concentration of phosphate, calcium, and magnesium in XCM1 and found that $\mathrm{XCM} 1$ with a lower concentration of magnesium (renamed as XCM2) is about 10 times as efficient as XCM1 (meanwhile, about 30 times stronger than MME). Thus, we established an inducing medium XCM2 which is preferred for T3 effector secretion in Xcc.
\end{abstract}

Keywords: inducing medium, type III effector secretion, Xanthomonas.

\section{Introduction}

The gram-negative bacterium Xanthomonas campestris pv. campestris $(X c c)$ is the causal agent of black rot disease, one of the most serious diseases of cruciferous crops worldwide (Alvarez, 2000). Over the last two decades, this pathogen has been used as a model bacterium for studying pathogen-plant interactions. Like many other important pathogenic bacteria, the pathogenicity of Xcc mainly relies on the type III secretion system (T3SS) that translocates so-called type III (T3) effector proteins into host cells. Generally the $\mathrm{T} 3$ effector proteins have a modular structure and the targeting signal resides in the first N-terminal 50 or 100 amino acids (Schechter et al., 2004). The T3SS of Xcc is encoded by $h r p$ (hypersensitive response and pathogenicity) genes whose expression is controlled by the key regulators HrpG and HrpX (Arlat et al., 1991; Huang et al., 2009). It has been demonstrated that in Xanthomonas spp. the expression of $h r p$ genes and most T3 effector genes is repressed in rich media but induced in minimal media and in plant tissues

Send correspondence to D.Q. Yu. Key Laboratory of Tropical Forest Ecology, Xishuangbanna Tropical Botanical Garden, Chinese Academy of Sciences, Kunming, 650223 Yunnan, China. E-mail: ydq@xtbg.ac.cn.

*These authors contributed equally to this work. 
(Schulte and Bonas, 1992b; Tsuge et al., 2002; Ryan et al., 2007; Wei et al., 2007).

The secretion of many $\mathrm{T} 3$ effectors is barely detectable in culture media, even with sensitive immunodetection (Collmer et al., 2002). To facilitate in-depth studies on T3SS and T3 effector proteins, it is crucial to establish a medium in which bacterial cells not only express their $h r p$ genes strongly but also secrete their T3 effector proteins efficiently. To date, four minimal media (MME, MMX, $\mathrm{XVM} 2$, and XOM2) have been used for expression induction of hrp genes in Xanthomonas spp., of which XOM2 and XVM2 have been developed for T3 secretion induction of $X$. oryzae pv. oryzae (Xoo) and X. campestris pv. vesicatoria (Xcv) respectively (Wengelnik et al., 1996; Tsuge et al., 2002; Ryan et al., 2007; Wei et al., 2007). However, the secretion efficiency of T3 effectors by Xcc in these media has not been determined. Moreover, no inducing medium for $\mathrm{T} 3$ effector secretion has been established in Xcc. In this work, based on a comparison of the four reported media, we investigated the effect of carbon and nitrogen sources, phosphate, calcium, and magnesium as well as $\mathrm{pH}$ value on T3 secretion efficiency of Xcc. Finally, we optimized a medium XCM2 that showed highly efficient induction of $\mathrm{T} 3$ effector secretion in Xcc.

\section{Materials and Methods}

\section{Bacterial strains, plasmids, and growth conditions}

Escherichia coli cells were grown in LB medium (Miller 1972) at $37^{\circ} \mathrm{C}$. Xcc wild-type strain 8004 (Daniels et al., 1984) and its derivative strains were cultivated at $28{ }^{\circ} \mathrm{C}$ in NYG rich medium $(3 \mathrm{~g} / \mathrm{L}$ yeast extract, $5 \mathrm{~g} / \mathrm{L}$ peptone, $20 \mathrm{~g} / \mathrm{L}$ glycerol, pH 7.0) (Daniels et al., 1984), or in minimal media MMX [23.8 mM glucose, $3.87 \mathrm{mM}$ sodium citrate, $15.1 \mathrm{mM}\left(\mathrm{NH}_{4}\right)_{2} \mathrm{SO}_{4}, 0.81 \mathrm{mM} \mathrm{MgSO}$, $23 \mathrm{mM} \mathrm{K}_{2} \mathrm{HPO}_{4}, 44 \mathrm{mM} \mathrm{KH}_{2} \mathrm{PO}_{4}, \mathrm{pH}$ 7.0] (Daniels et al., 1984), MME [20 mM sodium L-glutamate, $0.15 \mathrm{~g} / \mathrm{L}$ casamino acids, $7.57 \mathrm{mM}\left(\mathrm{NH}_{4}\right)_{2} \mathrm{SO}_{4}, 1 \mathrm{mM} \mathrm{MgSO}$, $60.34 \mathrm{mM} \mathrm{K}_{2} \mathrm{HPO}_{4}, 33.07 \mathrm{mM} \mathrm{KH}_{2} \mathrm{PO}_{4}, \mathrm{pH} 7.0$ ] (Ryan et al., 2007), XVM2 [10 mM sucrose, $10 \mathrm{mM}$ fructose, $0.01 \mathrm{mM}$ casamino acids, $10 \mathrm{mM}\left(\mathrm{NH}_{4}\right)_{2} \mathrm{SO}_{4}, 5 \mathrm{mM}$ $\mathrm{MgSO}_{4}, 0.16 \mathrm{mM} \mathrm{K} \mathrm{HPO}_{4}, 0.32 \mathrm{mM} \mathrm{KH} \mathrm{PO}_{4}, 20 \mathrm{mM}$ $\mathrm{NaCl}, 1 \mathrm{mM} \mathrm{CaCl}, 0.01 \mathrm{mM} \mathrm{FeSO}_{4}$, pH 6.7] (Wengelnik et al., 1996), XOM2[10 mM sodium L-glutamate, $1.8 \mathrm{~g} / \mathrm{L}$ xylose, $0.67 \mathrm{mM}$ methionine, $0.04 \mathrm{mM} \mathrm{MnSO}_{4}, 5 \mathrm{mM}$ $\mathrm{MgCl}_{2}, 14.7 \mathrm{mM} \mathrm{K}_{2} \mathrm{HPO}_{4}, \mathrm{pH}$ 6.5] (Tsuge et al., 2002), XCM1 $[20 \mathrm{mM}$ succinic acid, $0.15 \mathrm{~g} / \mathrm{L}$ casamino acids, $7.57 \mathrm{mM}\left(\mathrm{NH}_{4}\right)_{2} \mathrm{SO}_{4}, 1 \mathrm{mM} \mathrm{MgSO}{ }_{4}, 60.34 \mathrm{mM} \mathrm{K}_{2} \mathrm{HPO}_{4}$, $33.07 \mathrm{mM} \mathrm{KH}_{2} \mathrm{PO}_{4}, \mathrm{pH}$ 6.6], or XCM2 (as XCM1 but with $\left.0.01 \mathrm{mM} \mathrm{MgSO}_{4}\right)$. Antibiotics were used as described previously (Daniels et al., 1984).

\section{DNA manipulations}

Standard DNA manipulations were carried out as described previously (Sambrook et al., 1989). Enzymes sup- plied by Promega (USA) were used in accordance with the manufacturer's instructions. The conjugation between Xcc and $E$. coli strains was performed as described previously (Daniels et al., 1984).

\section{Construction of reporter plasmids and strains}

It is well known that $\mathrm{T} 3$ effectors normally have a modular structure, of which the secretion signal generally resides in the N-terminal 50 or 100 amino acids (Schechter et al., 2004). Our previous work demonstrated that $x o p X c c N$ of $X c c$ encodes a T3 effector (Jiang et al., 2008). In this work, we employed the reporter plasmid pLGUS0241 of XopXccN (Jiang et al., 2008) to study induction efficiency of T3 effector secretion. Another reporter plasmid, named pLGUS1553, was constructed by fusing the promoter and T3 signal sequences of the T3 effector gene $a v r A C_{X c c 8004}$ (Xu et al., 2008) with the promoterless $\beta$-glucuronidase (gusA) gene in the vector pLGUS (Jiang et al., 2008). These reporter plasmids were introduced from E. coli JM109 (Yanisch-Perron et al., 1985) into Xcc wild-type strain 8004 and the T3SS-deficient mutant strain $8004 \Delta \mathrm{hrcV}$ (data not published) by triparental conjugation using pRK2073 (Leong et al., 1982) as the helper plasmid, respectively. The yielding transconjugant strains 8004/pLGUS0241, 8004/pLGUS1553, and $8004 \Delta \mathrm{hrcV} / \mathrm{pLGUS} 0241$ were used for GUS activity assay.

The promoter and T3 signal sequences of $x o p X c c N$ were cloned into the EcoRI/BamHI sites of the reporter plasmid pJAG (Jiang et al., 2009), which was constructed previously and contains Plac promoter upstream of the multiple cloning sites and avrBs $1_{59-445}$ fused with the 5 ' end of 3xFLAG. The resulting plasmid named pJAG0241 was transferred into the strains 8004 and $8004 \Delta \mathrm{hrcV}$ by triparent conjugation, respectively. The yielding transconjugant strains 8004/pJAG0241 and 8004 $\Delta \mathrm{hrcV} / \mathrm{pJAG0241}$ were used for immunoblotting analysis.

\section{GUS activity assay}

The overnight cultured $X c c$ strains in NYG (pH 7.0) were resuspended to an optical density of $0.1(600 \mathrm{~nm})$ and cultivated in corresponding minimal medium with corresponding $\mathrm{pH}$ value for $20 \mathrm{~h}$. To determine the GUS activity of secreted proteins, the bacterial cells of $15-\mathrm{mL}$ culture for each strain was separated by centrifugation at $4000 \mathrm{~g}$ at $4{ }^{\circ} \mathrm{C}$ for $20 \mathrm{~min}$. The supernatant was filtered with a lowprotein-binding filter (Millipore Express Membrane, $0.22 \mu \mathrm{m}$, Millipore Corporation, USA). The cell-free supernatant concentrated to $500 \mu \mathrm{L}$ with centrifugal filter units (Amicon Ultra-15, $10 \mathrm{kD}$, Millipore, USA) was taken for GUS activity determination. For determination of the total GUS activity, $200 \mu \mathrm{L}$ culture for each strain was mixed with $40 \mu \mathrm{L}$ methylphenol and vortexed. The supernatant was then taken for GUS activity assay. The GUS ac- 
tivity assay was performed by measurement of the $\mathrm{OD}_{415}$ using $\rho$-nitrophenyl- $\beta$-D-glucuronide as substrate as described previously (Jefferson et al., 1986).

\section{Western immunoblotting analysis}

The supernatants of bacterial culture were extracted with the same conditions as in the GUS activity assay. For intracellular T3 effector analysis, after incubation for $20 \mathrm{~h}$, bacterial cells from 2-mL culture were precipitated and resuspended with $100 \mu \mathrm{L} / \mathrm{OD} . \mathrm{mL} 1$ western blue [6: $0.35 \mathrm{M}$ Tris- $\mathrm{HCl}(\mathrm{pH} 6.8), 10.28 \%$ SDS, 36\% glycerol, $0.6 \mathrm{M}$ DTT, $0.012 \%$ bromophenol blue, Promega]. Aliquots of $20 \mu \mathrm{L}$ were analyzed by immunoblotting. Anti-FLAG M5 polyclonal antibody and goat anti-rabbit IgG antibody (Sigma) were used as the primary and secondary antibodies, respectively. Reactions were visualized by WEST$\mathrm{ZOL}^{\mathrm{TM}}$ western blotting detection kit (iNtRON).

\section{Results}

\section{Comparison of four hrp-inducing media reported in Xanthomonas for T3 secretion efficiency of XcC}

As described above, it has been reported that the following four minimal media can significantly induce the expression of $h r p$ genes in Xanthomonas spp.: XVM2 in Xcv (Wengelnik et al., 1996), XOM2 in Xoo (Tsuge et al., 2002), MME and MMX in Xcc (Ryan et al., 2007; Wei et al., 2007). To compare the T3 secretion efficiency of Xcc in

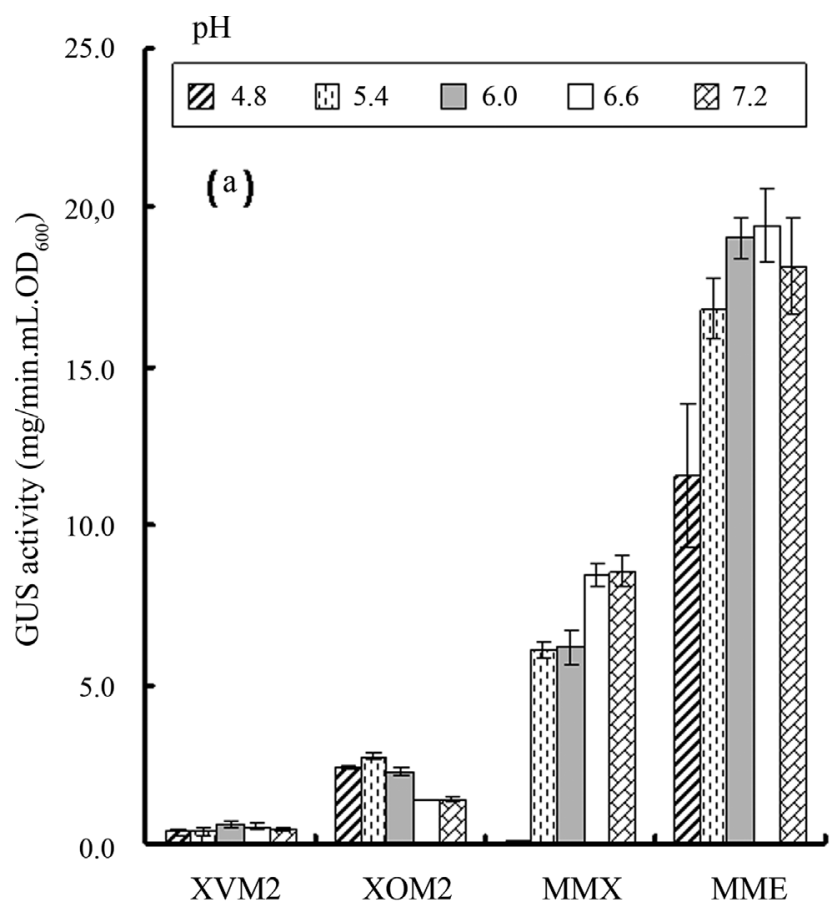

these four minimal media, we cultured the strain 8004/pLGUS0241 in the media and evaluated their GUS activities. Strain 8004/pLGUS0241 was constructed previously by introducing the reporter plasmid pLGUS0241 into the wild-type strain 8004 (Jiang et al., 2008). The reporter plasmid pLGUS0241 was created by fusing the promoter and T3 secretion signal sequence of the effector gene $x o p X c c N$ with the promoterless gusA gene cloned in the vector pLAFR6 (Huynh et al., 1989). The GUS activity produced by strain $8004 /$ pLGUS0241 can therefore reflect the production of the T3 effector XopXccN. As shown in Figure 1a, after 20 hours, the strain grown in MME medium produced 2.5- to 36-fold stronger total GUS activities than in other three media. GUS activities in the supernatants of XVM2 and XOM2 cultures were nearly undetectable (Figure 1b). Although some GUS activity was present in the supernatant of MMX culture, its level was significantly lower than that in the MME culture supernatant (Figure 1b). Simultaneously, we also assessed the influence of different $\mathrm{pH}$ values in the media on the GUS production of the strain and the result showed that the optimal $\mathrm{pH}$ value for the GUS production of the strain in MME medium was 6.6 (Figure 1). To verify the GUS activity in culture supernatant was not due to a leakage from the bacterial cells but secreted by T3SS, GUS activity in the culture supernatant of strain $8004 \Delta \mathrm{hrcV} / \mathrm{pLGUS0241}$, a strain constructed by introducing the reporter plasmid pLGUS0241 into the T3SS-deficient mutant $8004 \Delta \mathrm{hrcV}$, was assayed. No de-

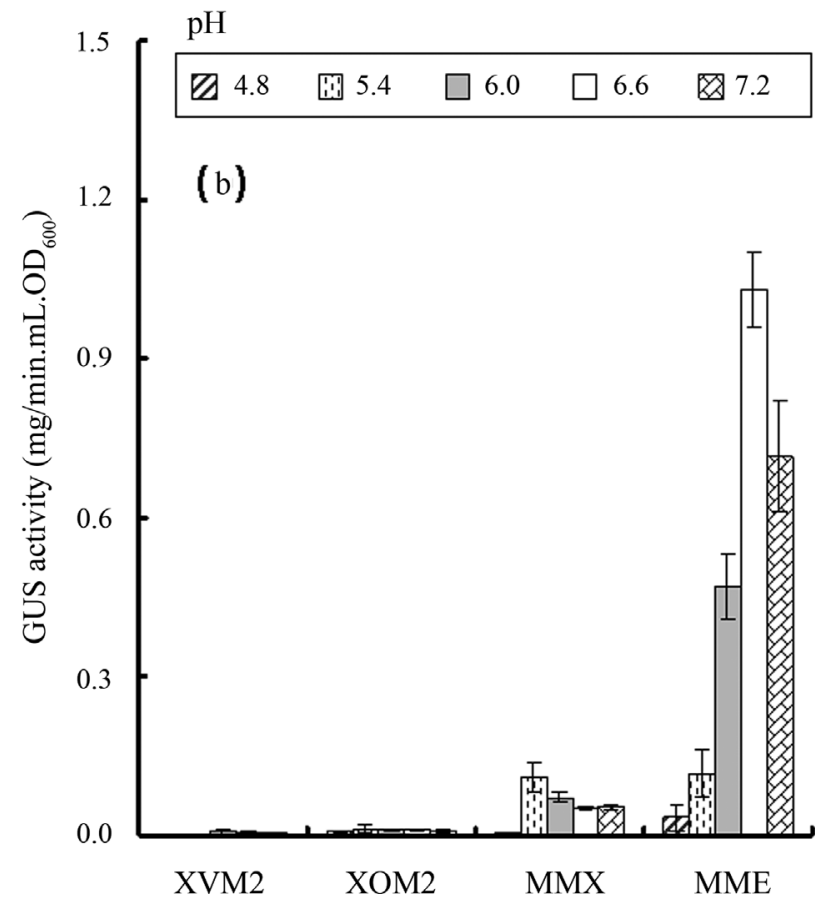

Figure 1 - The GUS activities in total cellular protein extracts (a) and in culture supernatants (b) of Xanthomonas campestris pv. campestris strain 8004/pLGUS0241 incubated for 20 hours in four minimal media (XVM2, XOM2, MMX, and MME) at different pH levels. Values given are the means and standard deviations of triplicate measurements. Data presented were from a representative experiment and similar results were obtained in two other independent experiments. 
tectable GUS activity was observed in the culture supernatants of $8004 \Delta \mathrm{hrcV} / \mathrm{pLGUS0241}$ grown in the four media for 20 hours (data not shown), suggesting that the above detected GUS activities in culture supernatants were not from a leakage of bacterial cells. Taking together, these results indicate that among the four media tested, MME is most efficient for induction of Xcc T3 expression and secretion. Therefore, MME was chosen for further study.

\section{Effect of amino acids in MME medium on T3 secretion efficiency}

The main carbon and nitrogen sources in MME medium are glutamate (Glu, an acidic amino acid) and casamino acids. To assess whether different amino acids have different effect on T3 secretion induction, we replaced glutamate in MME medium with alanine (Ala, a neutral amino acid), arginine (Arg, an alkaline amino acid), and aspartate (Asp, an acidic amino acid), respectively. After incubated in these different media for 20 hours, the GUS activities produced by strain 8004/pLGUS0241 were assayed. As shown in Table 1, the total GUS activities of strain 8004/pLGUS0241 grown in the four different media were not significantly different, while the supernatant GUS activities from the media with Glu (i.e. MME medium) and Ala were significantly stronger than those from the media with Arg and Asp. The supernatant GUS activities were not significantly different between the media with Glu and Ala (Table 1). These suggest that the different amino acids analyzed have a different influence on T3 secretion efficiency although they do not have a significant influence on the expression of the $\mathrm{T} 3$ effector tested.

It has been reported that sulfur-containing amino acids, especially methionine, are required for $h r p$ induction in $\mathrm{Xcv}$ (Schulte and Bonas, 1992a). To verify whether sulfur-containing amino acids have any effect on induction of Xcc T3 secretion, we compared the GUS activities of strain 8004/pLGUS0241 grown in MME medium with or without methionine (or cysteine). No significant difference was observed in either total GUS activities or supernatant GUS activities (Table 1). This result implies that sulfur-containing amino acids may have no effect on T3 expression and secretion in Xcc.

\section{Effect of carbon sources in MME medium on T3 secretion efficiency}

As described above, the carbon sources in the four media are different. To evaluate whether different carbon sources have an impact on T3 secretion efficiency in Xcc, we substituted the glutamate in MME with glucose, sucrose, fructose, glycerol, mannitol, and mannose, respectively. After incubation in these media for 20 hours, the GUS activities of strain 8004/pLGUS0241 were compared. As shown in Table 1, the results suggest that glutamate (MME) is the best among the tested carbon sources for T3 effector expression and secretion.
Since glutamic acid can enter the tricarboxylic acid (TCA) cycle through deamination, a query whether the substrates of the TCA cycle have an effect on T3 secretion was raised. To answer this question, we replaced glutamate in MME medium with $\alpha$-ketoglutarate, citrate, malate, fumarate, succinate, oxaloacetate, and pyruvate, respectively. Twenty hours after incubation, strain 8004/pLGUS0241 produced stronger total and supernatant GUS activities in the medium with glutamate than with all but one of the other carbon sources (Table 1). Approximately 3-fold stronger supernatant GUS activity in the medium with succinate was observed, as compared to the medium with glutamate (MME), although their total GUS activities were similar (Table 1). These results indicate that succinate is better than glutamate for induction of $\mathrm{T} 3$ effector secretion in Xcc.

Table 1 - GUS activity of strain 8004/pLGUS0241 in different growth conditions ${ }^{*}$.

\begin{tabular}{|c|c|c|}
\hline \multirow[t]{2}{*}{ Medium ingredients } & \multicolumn{2}{|c|}{$\beta$-Glucuronidase (GUS) activities } \\
\hline & Total & Supernatant \\
\hline MME & $21.748 \pm 1.364 \mathrm{a}$ & $1.158 \pm 0.148 \mathrm{a}$ \\
\hline $\mathrm{MME}+\operatorname{Met}(2 \mathrm{mg} / \mathrm{L})$ & $20.135 \pm 1.993 a$ & $1.068 \pm 0.118 \mathrm{a}$ \\
\hline $\mathrm{MME}+\operatorname{Met}(100 \mathrm{mg} / \mathrm{L})$ & $21.786 \pm 2.232 \mathrm{a}$ & $1.052 \pm 0.073 \mathrm{a}$ \\
\hline MME + Cys (2 mg/L) & $19.988 \pm 0.970 \mathrm{a}$ & $0.951 \pm 0.101 \mathrm{a}$ \\
\hline MME + Cys (100 mg/L) & $20.555 \pm 1.487 \mathrm{a}$ & $1.001 \pm 0.076 \mathrm{a}$ \\
\hline $\mathrm{A}+\mathrm{Ala}$ & $22.935 \pm 3.087 \mathrm{a}$ & $0.873 \pm 0.289 \mathrm{a}$ \\
\hline $\mathrm{A}+\mathrm{Arg}$ & $17.760 \pm 0.894 \mathrm{a}$ & $0.217 \pm 0.036 \mathrm{~b}$ \\
\hline$A+A s p$ & $20.109 \pm 0.487 \mathrm{a}$ & $0.250 \pm 0.044 \mathrm{~b}$ \\
\hline A + Glucose & $5.550 \pm 0.170 \mathrm{~b}$ & $0.370 \pm 0.029 b$ \\
\hline A + Sucrose & $3.299 \pm 0.049 b$ & $0.256 \pm 0.051 b$ \\
\hline A + Fructose & $4.207 \pm 0.255 b$ & $0.080 \pm 0.053 b$ \\
\hline A + Glycerol & $4.717 \pm 0.151 b$ & $0.121 \pm 0.014 \mathrm{~b}$ \\
\hline A + Mannitol & $13.793 \pm 0.262 b$ & $0.148 \pm 0.037 b$ \\
\hline A + Mannose & $6.073 \pm 0.082 b$ & $0.031 \pm 0.011 b$ \\
\hline$A+\alpha$-ketoglutarate & $13.561 \pm 0.911 b$ & $0.478 \pm 0.092 b$ \\
\hline A + Citrate & $14.593 \pm 0.683 b$ & $0.666 \pm 0.065 b$ \\
\hline A + Malate & $9.112 \pm 0.251 b$ & $0.374 \pm 0.094 \mathrm{~b}$ \\
\hline A + Fumarate & $11.325 \pm 0.513 b$ & $0.795 \pm 0.210 \mathrm{~b}$ \\
\hline A + Succinate & $19.680 \pm 0.691 \mathrm{a}$ & $3.472 \pm 0.090 \mathrm{~b}$ \\
\hline A + Oxaloacetate & $7.217 \pm 0.772 b$ & $0.627 \pm 0.068 b$ \\
\hline A + Pyruvate & $7.611 \pm 0.233 b$ & $0.370 \pm 0.029 b$ \\
\hline
\end{tabular}

* $\beta$-Glucuronidase (GUS) activity was determined after growth of $X$. campestris pv. campestris for $20 \mathrm{~h}$. Medium "A" has exactly the same composition of MME except for the absence of glutamate $(0.15 \mathrm{~g} / \mathrm{L}$ casamino acids, $7.57 \mathrm{mM}\left(\mathrm{NH}_{4}\right)_{2} \mathrm{SO}_{4}, 1 \mathrm{mM} \mathrm{MgSO}_{4}, 60.34 \mathrm{mM} \mathrm{K}_{2} \mathrm{HPO}_{4}$, and $33.07 \mathrm{mM} \mathrm{KH}_{2} \mathrm{PO}_{4}$ ). The $\mathrm{pH}$ of the variations of medium " $\mathrm{A}$ " was adjusted to 7.0 after the addition of amino acids or saccharides, as well as for the acids used as carbon source to medium "A". Data are the mean \pm standard deviation of triplicate measurements. The different letters in each data column indicate significant differences $(\mathrm{p}=0.01 ; t$-test $)$. 


\section{Effect of different concentrations of casamino acids on T3 secretion efficiency}

MME medium contains casamino acids as an organic nitrogen source. It has been reported that a high concentration of casamino acids can suppress the induction of hrp expression in $X c v$ (Schulte and Bonas, 1992a). To optimize the concentration of casamino acids for high T3 secretion efficiency, we examined the effect of various concentrations of casamino acids on the induction of T3 effector secretion. Since succinate is better than glutamate for induction of T3 effector secretion, for a better test we used a modified MME medium in which the carbon source glutamate was replaced with succinate and its $\mathrm{pH}$ value was adjusted to 6.6 with $\mathrm{HCl}$. As shown in Figure 2, the total GUS activities produced by strain 8004/pLGUS0241 decreased along with the increase of the concentrations of casamino acids. However, the GUS activity in the supernatant of the culture with $0.15 \mathrm{~g} / \mathrm{L}$ casamino acids was 2 - to 3 -fold stronger than other concentrations including the lower concentration of $0.075 \mathrm{~g} / \mathrm{L}$ (Figure 2). This result suggests that an optimal concentration of casamino acids for $\mathrm{T} 3$ effector secretion is about $0.15 \mathrm{~g} / \mathrm{L}$, which is the original concentration in MME medium. Based on all of the above findings, we have designed a medium for T3 secretion induction in $X c c$ by modification of MME medium, in which the carbon source glutamate is substituted by succinate and $\mathrm{pH}$ value is

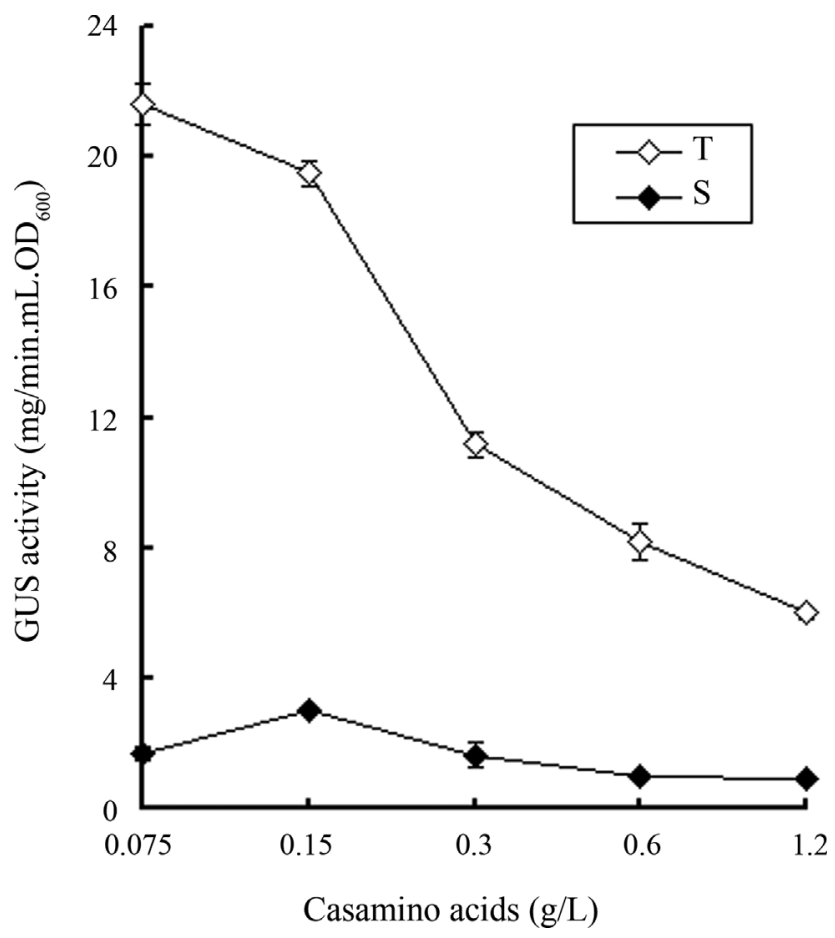

Figure 2 - Effect of different concentrations of casamino acids on the GUS activities in total cellular protein extracts $(\mathrm{T})$ and in culture supernatants (S) of Xanthomonas campestris pv. campestris strain 8004/pLGUS0241 grown in medium XCM1 for 20 hours. Values given are the means and standard deviations of triplicate measurements. Data presented were from a representative experiment and similar results were obtained in two other independent experiments. adjusted to 6.6. We have named this modified MME medium as XCM1.

\section{Additional evidences that XCM1 induces the secretion of $\mathrm{T} 3$ effectors efficiently}

To evaluate whether the medium XCM1 is also practical for efficient secretion induction of other T3 effectors in Xcc, we randomly selected another T3 effector, $\mathrm{AvrAC}_{\mathrm{Xcc8004}}$ (Xu et al., 2008), to test its secretion induction in XCM1. A reporter plasmid named pLGUS1553 was constructed by fusing the promoter and T3 signal of the effector gene $a v r A C_{X c c 8004}$ with the promoterless gus $A$ gene cloned in pLAFR6 and introduced into the wild-type strain 8004 (see above for details). The supernatant GUS activities of the resulting strain 8004/pLGUS1553 grown in XVM2, XOM2, MMX, MME, and XCM1 were compared. The result showed that XCM1 induced significantly stronger GUS activity than all other media (Figure 3), indicating that XCM1 medium is also efficient for the secretion induction of the $\mathrm{T} 3$ effector protein $\mathrm{AvrAC}_{\mathrm{Xcc8004}}$.

We also performed a western immunoblotting analysis to verify the secretion induction efficiency of the T3 effector XopXccN in XCM1 medium (see above for details). The results showed that strain 8004/pJAG0241 grown in XCM1 and MME produced similar, stronger intracellular XopXccN protein bands compared to other three media (Figure 4a). Moreover, a stronger XopXccN protein band could be seen for the culture supernatant of XCM1 medium compared to MME medium, while no visible XopXccN protein band could be detected for the culture supernatants of other three media (Figure $4 b$ ). These results suggest that XCM1 medium is more efficient for the secretion of XopXccN effector. In the study, a strain deficient in T3 secretion, named 8004 $\Delta \mathrm{hrcV} / \mathrm{pJAG} 0241$, was included.

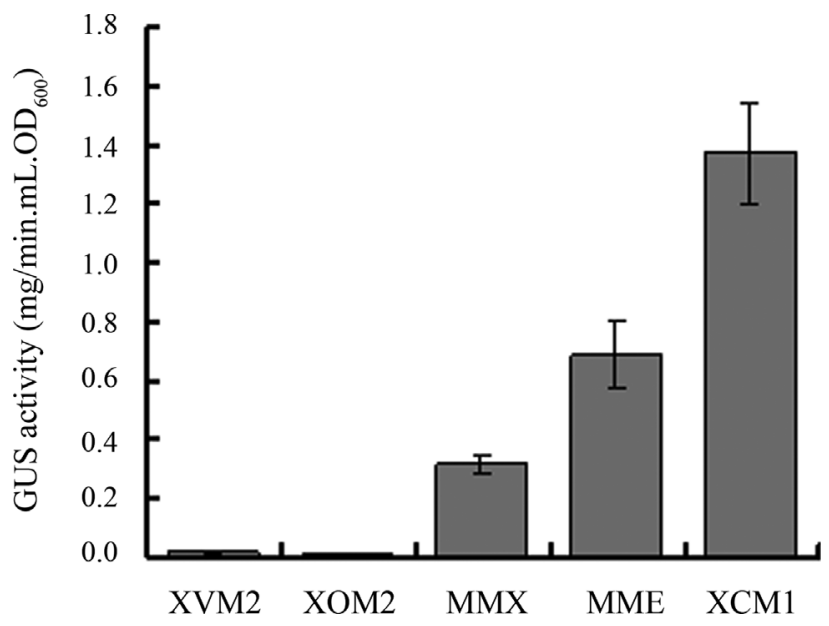

Figure 3 - The GUS activities in culture supernatants of Xanthomonas campestris pv. campestris strain 8004/pLGUS1553 incubated for 20 hours in five minimal media (XVM2, XOM2, MMX, MME, and XCM1). Values given are the means and standard deviations of triplicate measurements. Data presented were from a representative experiment and similar results were obtained in two other independent experiments. 
XCM1 MME $\quad$ MMX XOM2 XVM2

(a)

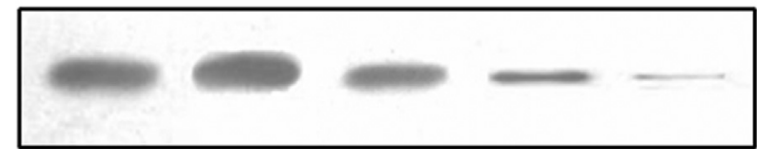

(b)

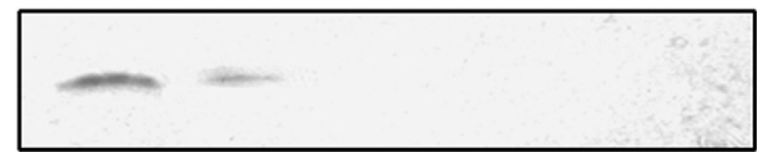

Figure 4 - Western immunoblotting analysis of the T3 effector XopXccN of Xanthomonas campestris pv. campestris. The bacterial cells of strain 8004/pJAG0241 were overnight cultivated in NYG (pH 7.0) and resuspended to an optical density of $0.1(600 \mathrm{~nm})$ in $100 \mathrm{~mL}$ of corresponding minimal media (XCM1, MME, MMX, XOM2, and XVM2). Equal amounts of intercellular protein extracts (a) and culture supernatants (b) were analyzed by SDS-PAGE and immunoblotting using the anti-FLAG M5 polyclonal antibody and goat anti-rabbit IgG antibody (Sigma) as the primary and secondary antibodies, respectively. Reactions were visualized by WEST-ZOL ${ }^{\mathrm{TM}}$ western blotting detection kit (iNtRON). The experiment was repeated twice and similar results were obtained.

As expected, no secreted XopXccN protein was detected in the supernatants of bacterial cultures grown in all of the five media, while intracellular XopXccN protein could be detected (data not shown). These further confirmed that $\mathrm{XCM} 1$ medium is efficient for secretion induction of $\mathrm{T} 3$ effector proteins in $X c c$.

\section{Effect of phosphate, calcium, and magnesium on T3 secretion efficiency}

It has been reported that phosphate, calcium, and magnesium affect $\mathrm{T} 3$ expression and secretion in some ani- mal pathogenic bacteria (Williams et al., 1998; Miao et al., 2002; Duong et al., 2007; Vilches et al., 2009; Anderson et $a l ., 2010)$. We realized that an important difference among the five minimal media is the concentrations of phosphate (XCM1, MME, MMX, and XOM2 but not XVM2 contain high concentrations of phosphate) (See details in Materials and Methods). The effect of phosphate on T3 secretion efficiency in Xcc should be further investigated. After incubated in XCM1 with different concentrations of phosphate $\left(10-, 2-, 1-, 10^{-1}-, 10^{-2}\right.$-fold, while 1-fold means the original concentration of phosphate: $60.34 \mathrm{mM} \mathrm{K}_{2} \mathrm{HPO}_{4}, 33.07 \mathrm{mM}$ $\mathrm{KH}_{2} \mathrm{PO}_{4}$ ) for 20 hours, the GUS activities produced by strain 8004/pLGUS0241 were assayed. The results showed that the original 1-fold concentration of phosphate has the best induction efficiency for secretion of XopXccN (data not shown).

It is interesting that XVM2 contains calcium and was proved to be the poorest medium for Xcc T3 secretion (Figure 1). It has been known for a long time that calcium inhibits T3 secretion in bacterial pathogens that infect animals (Williams et al., 1998). The effect of calcium on T3 secretion efficiency in Xcc was further surveyed. Twenty hours after incubation in XCM1 without or with different concentrations of calcium chloride $(0.5 \mathrm{mM}, 1 \mathrm{mM}, 2 \mathrm{mM}, 3 \mathrm{mM})$, the GUS activities of strain 8004/pLGUS0241 were assayed. The GUS activity in the supernatant of the culture without $\mathrm{CaCl}_{2}$ was about 3 -fold stronger than that with different $\mathrm{CaCl}_{2}$ concentrations. This result implies that calcium may repress T3 secretion in Xcc (Figure 5a).

In addition, it was reported that magnesium modulates (activates or represses) T3 expression and even secre-
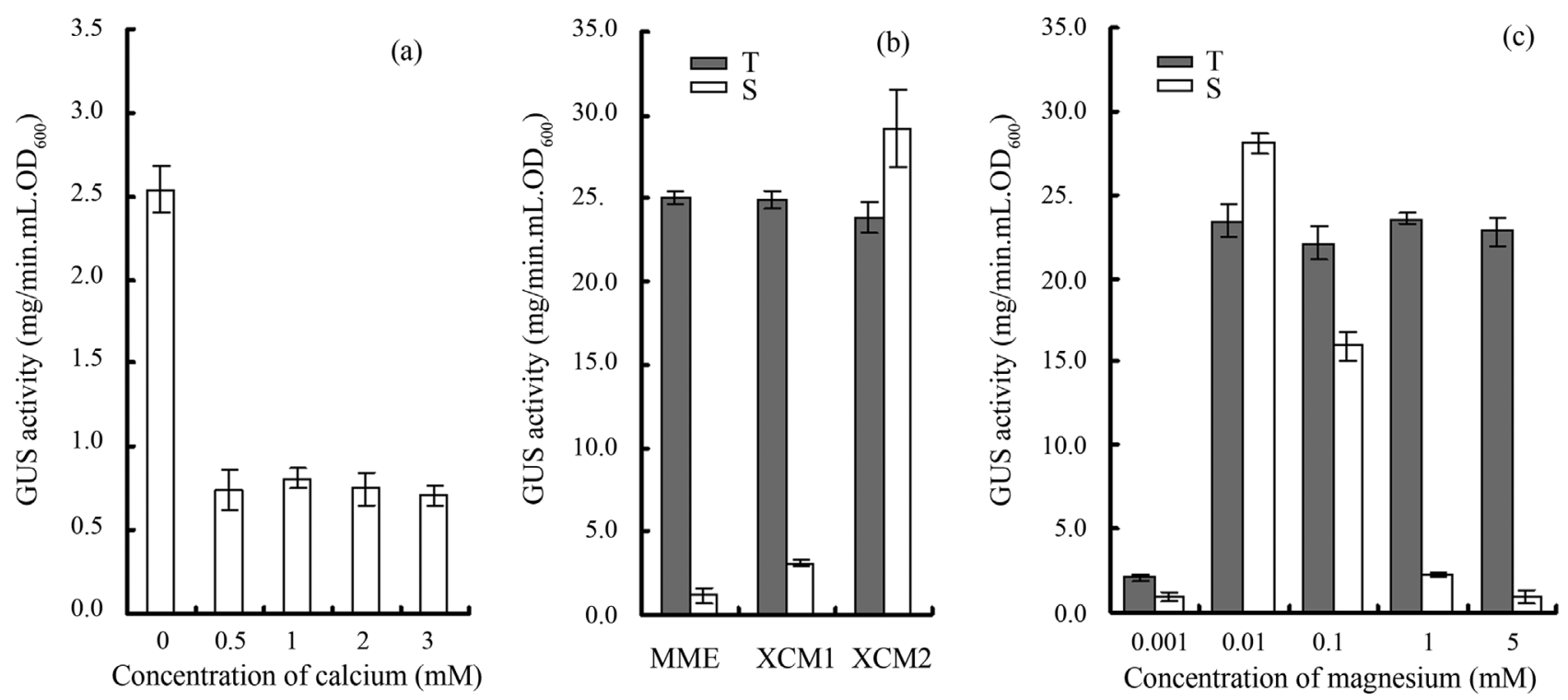

Figure 5 - Effect of different concentrations of calcium and magnesium on the $\beta$-Glucuronidase (GUS) activities of Xanthomonas campestris pv. campestris strain 8004/pLGUS0241. (a) The supernatant GUS activities produced by the strain grown in XCM1 medium with different CaCl 2 concentrations for 20 hours. (b) The GUS activities in total cellular protein extracts (T) and culture supernatants (S) produced by the strain grown for 20 hours in different media. (c) The GUS activities in total cellular protein extracts (T) and culture supernatants (S) produced by the strain grown for 20 hours in XCM1 medium with different $\mathrm{MgSO}_{4}$ concentrations. Values given are the means and standard deviations of triplicate measurements. Data presented were from a representative experiment and similar results were obtained in two other independent experiments. 
tion in some animal bacterial pathogens (Williams et al., 1998; Miao et al., 2002; Vilches et al., 2009; Anderson et al., 2010). We also notice that each of the five media tested (XCM1, MME, MMX, XVM2, and XOM2) contains a different concentration of magnesium. To verify whether the concentration of magnesium affects Xcc T3 secretion efficiency, we compared the GUS activities of strain 8004/pLGUS0241 incubated in MME (contains $1 \mathrm{mM}$ $\mathrm{MgSO}_{4}$ ), XCM1 (contains $1 \mathrm{mM} \mathrm{MgSO}$ ), and XCM2 (contains $0.01 \mathrm{mM} \mathrm{MgSO}$, see below). The strain produced similar total GUS activities in the three media; however, it produced 10-30 fold supernatant GUS activity in $\mathrm{XCM} 2$, compared to XCM1 and MME (Figure 5b). The GUS activities produced by strain 8004/pLGUS0241 incubated in XCM1 with different $\mathrm{MgSO}_{4}$ concentrations were further tested. As shown in Figure 5c, although no significant difference in total GUS activities was observed among the $\mathrm{MgSO}_{4}$ concentrations of $0.01,0.1,1$, and $5 \mathrm{mM}$, extremely high supernatant GUS activity was found in the $0.01 \mathrm{mM} \mathrm{MgSO}_{4}$ concentration. These results suggest that an optimal concentration of $\mathrm{MgSO}_{4}$ for $\mathrm{T} 3$ effector secretion is about $0.01 \mathrm{mM}$. Therefore, we further modified XCM1 by adjusting its $\mathrm{MgSO}_{4}$ concentration from $1 \mathrm{mM}$ to $0.01 \mathrm{mM}$, and named this modified XCM1 medium as $\mathrm{XCM} 2$.

Taken together, we have established a medium named as XCM2 for T3 secretion induction in Xcc by modification of MME medium, in which the carbon source glutamate is substituted by succinate, concentration of $\mathrm{MgSO}_{4}$ is optimized to $0.01 \mathrm{mM}$, and $\mathrm{pH}$ value is adjusted to 6.6 .

\section{Discussion}

The secretion of many bacterial $\mathrm{T} 3$ effectors is barely detectable in culture media, even with sensitive immunodetection (Collmer et al., 2002); therefore, a high efficient inducing medium is crucial for identification of novel T3 effectors. XCM2 established here exhibits significantly higher efficiency for Xcc T3 effector secretion than MME, although they showed similar induction efficiency for T3 effector expression (Figures 3, 4, and 5, Table 1). The western immunoblotting analysis results showed that the secretion of XopXccN could be detected clearly in XCM1, but weakly in MME and undetectable in all of the other media tested (Figure 4b), suggesting that the about 3 -time inducing efficiency attributed by succinate is important for detection of T3 effector in Xcc. Moreover, XCM2 displayed about 10 -fold and 30 -fold stronger inducing efficiency than XCM1 and MME, respectively (Figure 5), indicating that XCM2 is beneficial for secretion induction of $X c c$ T3 effectors. Additionally, the secretion efficiencies of the effector XopXccN in several Xcc strains including CN07 and CN11 (He et al., 2007) are all distinctly higher in $\mathrm{XCM} 2$, compared to MME (data not shown), suggesting that XCM2 could be widely used for different Xcc strains. This optimized medium will provide a powerful tool for
T3S effector identification and functionality studies in Xcc and hence contributes to the basic concepts and understanding of molecular pathogenesis of phytopathogen.

\section{Acknowledgments}

This work was supported by the National Natural Science Foundation of China (30970085) and the National Special Grant for Transgenic Researches (2009ZX08009038B).

\section{References}

Alvarez AM (2000) Black rot of crucifers. In: Slusarenko AJ, Fraser RSS, van Loon LC (eds) Mechanisms of Resistance to Plant Diseases. Springer, Dordrecht, pp 21-52.

Anderson GG, Yahr TL, Lovewell RR, O'Toole GA (2010) The Pseudomonas aeruginosa magnesium transporter MgtE inhibits transcription of the type III secretion system. Infect Immun 78:1239-1249.

Arlat M, Gough C, Barber C, Boucher C, Daniels M (1991) Xanthomonas campestris contains a cluster of hrp genes related to the larger $h r p$ cluster of Pseudomonas solanacearum. Mol Plant-Microbe Interact 4:593-601.

Collmer A, Lindeberg M, Petnicki-Ocwieja T, Schneider DJ, Alfano JR (2002) Genomic mining type III secretion system effectors in Pseudomonas syringae yields new picks for all TTSS prospectors. Trends Microbiol 10:462-469.

Daniels MJ, Barber CE, Turner PC, Cleary WG, Sawczyc MK (1984) Isolation of mutants of Xanthomonas campestris pv. campestris showing altered pathogenicity. Gen Microbiol 130:2447-2455.

Duong N, Osborne S, Bustamante VH, Tomljenovic AM, Puente JL, Coombes BK (2007) Thermosensing coordinates a cisregulatory module for transcriptional activation of the intracellular virulence system in Salmonella enterica serovar Typhimurium. J Biol Chem 282:34077-34084.

He YQ, Zhang L, Jiang BL, Zhang ZC, Xu RQ, Tang DJ, Qin J, Jiang W, Zhang X, Liao J, Cao JR, Zhang SS, Liang X.X, Wei ML, Lu GT, Feng JX, Chen B, Cheng J, Tang JL (2007) Comparative and functional genomics reveals genetic diversity and determinants of host specificity among reference strains and a large collection of Chinese isolates of the phytopathogen Xanthomonas campestris pv. campestris. Genome Biology 8:R218.

Huang DL, Tang DJ, Liao Q, Li XQ, He YQ, Feng JX, Jiang BL, Lu GT, Tang JL (2009) The Zur of Xanthomonas campestris is involved in hypersensitive response and positively regulates the expression of the $h r p$ cluster via $h r p X$ but not $h r p G$. Mol Plant-Microbe Interact 22:321-329.

Huynh TV, Dahlbeck D, Staskawicz BJ (1989) Bacterial blight of soybean: regulation of a pathogen gene determining host cultivar specificity. Science 245:1374-1377.

Jefferson R, Burgess S, Hirsh D (1986) $\beta$-Glucuronidase from Escherichia coli as a gene-fusion marker. Proc Natl Acad Sci USA 83:8447-8451.

Jiang BL, He YQ, Cen WJ, Wei HY, Jiang GF, Jiang W, Hang XH, Feng JX, Lu GT, Tang DJ, Tang JL (2008) The type III secretion effector XopXccN of Xanthomonas campestris pv. campestris is required for full virulence. Res Microbiol 159:216-220. 
Jiang W, Jiang BL, Xu RQ, Huang JD, Wei HY, Jiang GF, Cen WJ, Liu J, Ge YY, Li GH, Su LL, Hang XH, Tang DJ, Lu GT, Feng JX, He YQ, Tang JL (2009) Identification of six type III effector genes with the PIP box in Xanthomonas campestris pv. campestris and five of them contribute individually to full pathogenicity. Mol Plant-Microbe Interact 22:1401-1411.

Leong SA, Ditta GS, Helinski DR (1982) Heme biosynthesis in Rhizobium. Identification of a cloned gene coding for deltaaminolevulinic acid synthetase from Rhizobium meliloti. J Biol Chem 257:8724-8730.

Miao EA, Freeman JA, Miller SI (2002) Transcription of the $\mathrm{SsrAB}$ regulon is repressed by alkaline $\mathrm{pH}$ and is independent of PhoPQ and magnesium concentration. J Bacteriol 184:1493-1497.

Miller JH (1972) Experiments in Molecular Genetics. Cold Spring Harbor, New York.

Ryan RP, Fouhy Y, Lucey JF, Jiang BL, He YQ, Feng JX, Tang JL, Dow JM (2007) Cyclic di-GMP signalling in the virulence and environmental adaptation of Xanthomonas campestris. Mol Microbiol 63:429-442.

Sambrook J, Fritsch EF, Maniatis T (1989) Molecular Cloning: A Laboratory Manual. Cold Spring Harbour Laboratory Press, New York

Schechter LM, Roberts KA, Jamir Y, Alfano JR, Collmer A (2004) Pseudomonas syringae type III secretion system targeting signals and novel effectors studied with a Cya translocation reporter. J Bacteriol 186:543-555.

Schulte R, Bonas U (1992a) A Xanthomonas pathogenicity locus is induced by sucrose and sulfur-containing amino acids. Plant Cell 4:79-86.

Schulte R, Bonas U (1992b) Expression of the Xanthomonas campestris pv. vesicatoria hrp gene cluster, which determines pathogenicity and hypersensitivity on pepper and tomato, is plant inducible. $\mathrm{J}$ Bacteriol 174:815-823.
Tsuge S, Furutani A, Fukunaka R, Oku T, Tsuno K, Ochiai H, Inoue $\mathrm{Y}$, Kaku H, Kubo Y (2002) Expression of Xanthomonas oryzae pv. oryzae hrp genes in XOM2, a novel synthetic medium. J Gen Plant Pathol 68:363-371.

Vilches S, Jimenez N, Tomas JM, Merino S (2009) Aeromonas hydrophila $\mathrm{AH}-3$ type III secretion system expression and regulatory network. Appl Environ Microbiol 75:6382-6392.

Wei K, Tang DJ, He YQ, Feng JX, Jiang BL, Lu GT, Chen B, Tang JL (2007) $h p a R$, a putative marR family transcriptional regulator, is positively controlled by HrpG and HrpX and involved in the pathogenesis, hypersensitive response, and extracellular protease production of Xanthomonas campestris pathovar campestris. J Bacteriol 189:2055-2062.

Wengelnik K, Marie C, Russel M, Bonas U (1996) Expression and localization of HrpA1, a protein of Xanthomonas campestris pv. vesicatoria essential for pathogenicity and induction of the hypersensitive reaction. J Bacteriol 178:1061-1069.

Williams AW, Straley SC (1998) YopD of Yersinia pestis plays a role in negative regulation of the low-calcium response in addition to its role in translocation of Yops. J Bacteriol 180:350-358.

Xu RQ, Blanvillain S, Feng JX, Jiang BL, Li XZ, Wei HY, Kroj T, Lauber E, Roby D, Chen B, He YQ, Lu GT, Tang DJ, Vasse

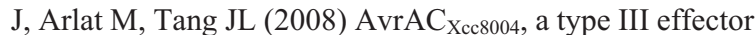
with a leucine-rich repeat domain from Xanthomonas campestris pathovar campestris confers avirulence in vascular tissues of Arabidopsis thaliana ecotype Col-0. J Bacteriol 190:343-355.

Yanisch-Perron C, Vieira J, Messing J (1985) Improved M13 phage cloning vectors and host strains: nucleotide sequences of the M13mp18 and pUC19 vectors. Gene 33:103-119.

All the content of the journal, except where otherwise noted, is licensed under a Creative Commons License CC BY-NC. 